\title{
A Closed-Loop Adaptive System for Command and Control
}

\author{
Tjerk de Greef ${ }^{1}$, Henryk Arciszewski ${ }^{2}$ \\ ${ }^{1}$ Department of Human in Control, TNO Human Factors, \\ PO BOX 23, 3769 ZG Soesterberg, The Netherlands \\ 2 Department of C2 and Information, TNO Information \& Operations, \\ PO BOX 23, 3769 ZG Soesterberg, The Netherlands \\ \{Tjerk.deGreef, Henryk.Arciszewski\}@tno.nl
}

\begin{abstract}
On Navy ships, technological developments enable crews to work more efficiently and effectively. However, in such complex, autonomous, and information-rich environments a competition for the users' attention is going on between different information items, possibly leading to a cognitive overload. This overload originates in the limitations of human attention and constitutes a well-known and well-studied bottleneck in human information processing. The concept of adaptive automation promises a solution to the overwhelmed operator by shifting the amount of work between the human and the system in time, while maintaining a high level of situation awareness. One of the most critical challenges in developing adaptive human-machine collaboration concerns the design of a trigger mechanism. This paper discusses and evaluates a number of possible triggers for the usage in closed-loop adaptive automation from the perspective of command and control.
\end{abstract}

\section{Introduction}

On navy ships, technological developments enable crews to work more efficiently and effectively. However, in such complex, autonomous, and information-rich environments, task demands may exceed the users' limited cognitive resources, possibly leading to a state of overload. This constitutes a well-known and well-studied bottleneck in human information processing. Besides the technical developments, the manning reduction initiatives of many navies cause a further increase of the workload of crew.

It is suggested that the adaptive automation paradigm may present a good equilibrium between task demands and the available cognitive resources. Adaptive automation (AA) takes as its starting point that the division of labor between man and machine should not be static but dynamic in nature. It is based on the conception of actively supporting the operator only at those moments in time when human performance in a system needs support to meet operational requirements [1]. Some argue that the appliance of adaptive automation enhances performance, reduce workload, and improve situation awareness [2]. Since 1988 various empirical studies have proven beneficial effects of the concept of adaptive automation [3] [4] [5] [6] [7] [8].

One of the challenging factors in the development of a successful adaptive automation concept concerns the question of when changes in level of automation must be ef- 
fectuated. With respect to methods of invocation, one of the most critical challenges facing designers of adaptive systems is how to effectively switch between the levels and/or modes of operation. The definition of augmented cognition extends the AA paradigm by explicitly stating the symbolic integration of man and machines in a closed-loop system whereby the operator's cognitive state and the operational context are to be detected by the system [9].

Workload generally is the key concept to invoke such a change but this concept is used in the broadest sense only. The measurement of workload is again much debated and we would like to elucidate the relationship between workload, task demands, and performance. Fig 1 shows the relationship between these three variables.

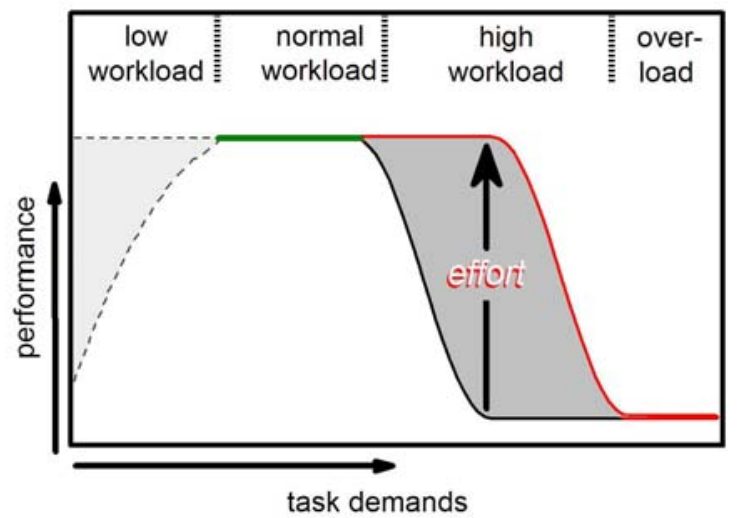

Fig. 1. The relation between task demands, performance, and workload (taken from [10]).

It shows that an operator can experience different levels of workload dependent on the demands of a task. It also shows that the performance does not necessarily decline as the operator experiences a high workload. We can cope with changing conditions without decreasing our performance or getting into a state of overload by putting more energy into it thereby making ourselves an adaptive system. The difference between maintaining the level of performance and an increased workload is referred to as the effort of the operator.

The majority of the studies have facilitated allocations based on critical events, performance models, or physiological measurements, all with their benefits and pitfalls. The critical-event method uses specific tactical events as triggers and suffers from the fact that this method is insensitive to the operator's available resources. Performance models have been argued as being totally reactive and unlikely to craft a comprehensive database [11]. The psychophysical approach uses deviations in EEG power bands, heart rate variability, respiration, galvanic skin response, and brain activity as trigger mechanisms. These studies suggest that it is indeed possible to obtain indices of one's brain activity and use that information to drive an adaptive automation system to improve performance and moderate workload. There are, however, still many critical conceptual and technical issues that must be overcome before systems such as these can move from the laboratory to the field [12]. 
None of the studies have reported on the application of AC in the actual operational field such as command and control (C2), although a number of studies have generalized a number of tasks from C2 for experimental purposes. These studies demonstrate enhanced performance, a reduction of workload, and improved situation awareness, though none report on the utilization of AA in an operational settings. Manning reduction initiatives and technological developments confront warfare officers with more information and the risk of overload and underload seems eminent. This study centralizes on the application of AA in the field of C2, particularly on the question which triggers are useful in this area of operation. This study abstains from the usage of psychophysical triggers since research by Veltman \& Jansen [10] report on the difficulties of such measure in the field of C2. This study centralizes on the operational field and the question which factors could contribute as indicators of overload. We gathered data from the same as study Veltman \& Jansen [10] and demonstrate that performance modeling provides one indicator, but should be extended with clever usage of environmental data. This paper suggests that creating triggers based on environmental data in combination with performance measurement might deliver a reliable trigger for applying the concept of AA.

Section two reports the theory on the performance model and section three validates the performance model with data from the study by Veltman \& Jansen [10]. Chapter four demonstrates how environmental data from the field of C2 might be used as an indication of workload and section five learns how pro-active behavior can be incorporated. The last section concludes this paper.

\section{Performance modeling}

As stated previously, we focus on a performance measure and state that the measure of operator performance provides an indication of the cognitive state. Specifically, the acknowledgement time (AT) of the operator on signals from the combat management system (CMS) is the primary base of the performance measure. Accordingly, a performance-decrease is regarded as a situation where the operator fails to cope with the situation and the operation requires assistance of the system. These situations arise due to temporal limited human information processing capacity.

The chosen feedback approach works only under the premise that the system signals the operator intelligently. Hence, the operator is prompted only when true information is available and true information should be regarded according to the definition of Shannon \& Weaver [13] as the reduction of uncertainty. This premise is achieved through the separation of the user and system understanding of the surrounding world (see fig. 2). Both user and system use its own particular reasoning mechanisms to create such an understanding. The system reasons through artificial intelligence mechanisms using sensory information while the human applies cognitive resources to craft such an understanding. The information elements (e.g. track) are present in each view though their attributes (e.g. speed, height) may differ. Each element can be compared for differences. For instance, the identities assigned by system and user may not be the same. Accordingly, the CMS signals information to the operator only when the system finds new evidence that adjusts the current understand- 
ing. The operator on the other hand, can acknowledge signaled information by explicitly stating its understanding of the situation and the CMS stores this opinion in the user world view (see fig. 2).
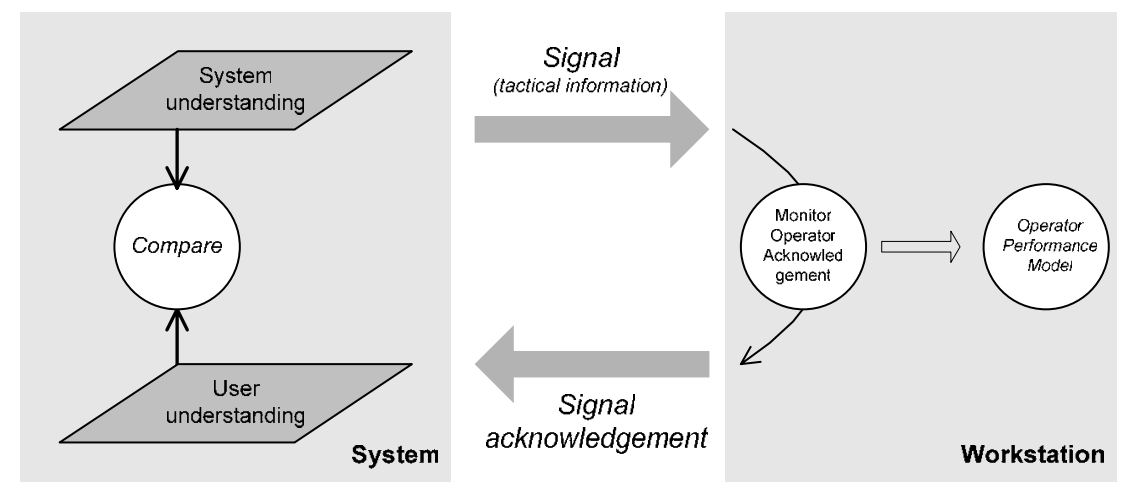

Fig. 2. The workstation receives information from the system and communicates the operator's understanding of the situation opinion to the system for storage in the user understanding space. The operator performance is calculated real-time and is based on the acknowledgement of the operator responses to signals

The workstation has two responsibilities. First, the workstation ought to keep the information element within the system synchronized with the user's state of mind. Secondly the workstation must measure the user's cognitive state. The first responsibility is achieved through the passing of the user's understanding, and the second responsibility is accomplished by comparing the acknowledgement times against an operator performance model (OPM). A significant increase in the AT corresponds with a drop in performance and with a state of overload, which on its turn leads to increased assertiveness of the workstation.

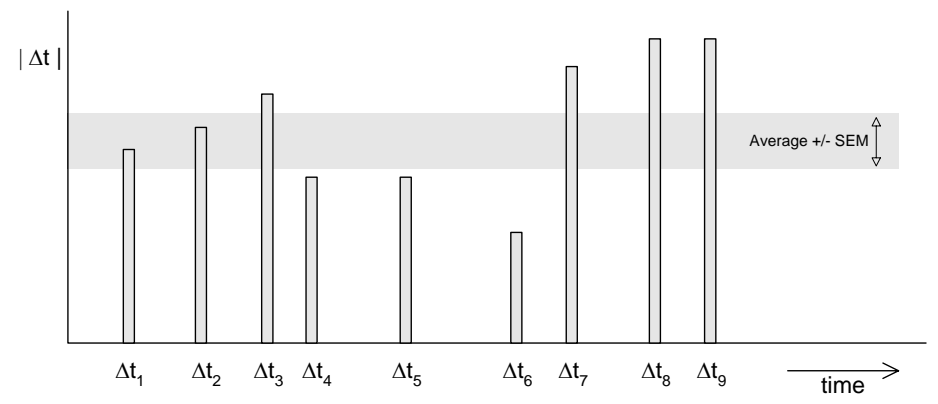

Fig. 3. Detecting an increase in acknowledgement time means a decrease in performance

The OPM is crafted in a learning phase from the average AT of the user to signals of the CMS regarding elements that are signaled. The OPM determines the average response (AVG) time and the standard error of means (SEM). Standard errors of means are important because they reflect how much sampling fluctuation, that is the extent 
to which a statistic takes on different values with different samples, a statistic will show.

As stated, the OPM is crafted in a learning phase. Once the OPM is properly trained, the model defines a bandwidth of the average AT +/- SEM (see fig 3). Any AT that is larger than the average AT + SEM is a trigger to increase the authority of the system.

\section{Validation}

This section presents a preliminary validation of the described performance model for three reasons. First, although sensitive to momentary changes, the performance modeling approach is criticized as being totally reactive. Secondly it is unlikely that a comprehensive database could be established [11]. Thirdly, we surmise that the described signaling paradigm might demonstrate a low sample rate, since signaling is only done in those cases where reduction of uncertainty is achieved. Prior to running an experiment with the performance model, we applied the model using data from another experiment and looked into these issues.

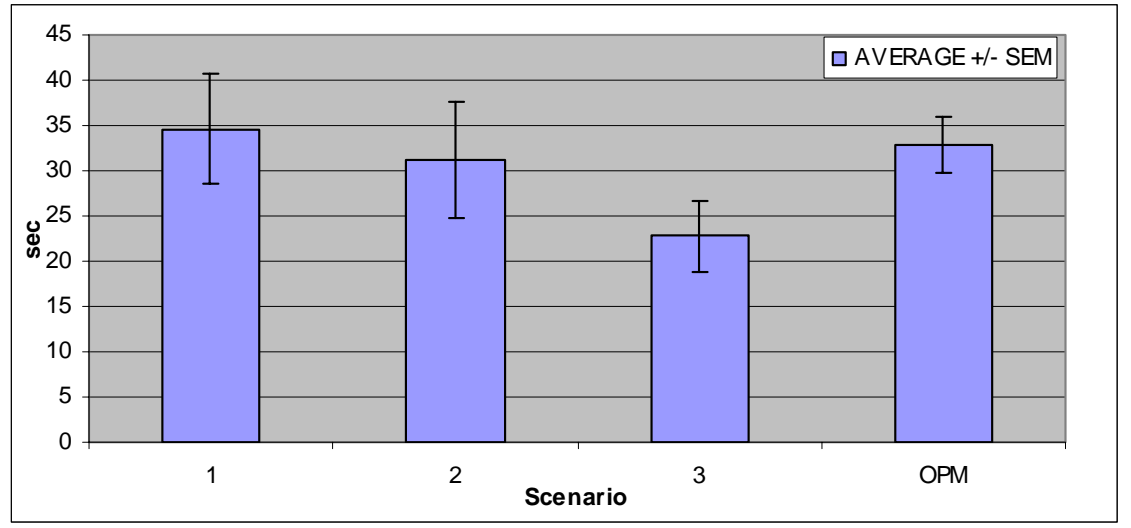

Fig. 4. The average acknowledge time and standard error of means per scenario for one WO

During the evaluation of a novel workstation [14] with eight warfare officers (WO) of the Royal Netherlands Navy, acknowledgement-times were recorded. The evaluation was arranged to prove the power of the workstation. Mitigation strategies were not available. The workstation was designed around a simulation environment that fed environmental and entity data to a ship model containing sensors, weapons and a highly automated combat management system (CMS). The information flow from the CMS communicates information in the form of tracks that include position, velocity, identity, and threat-level. The CMS is able to build and retrieve track information thanks to its range of sensors and reasoning functionality. When new information regarding a track becomes available, the CMS signals this information to the workstation only when the user has a different understanding of the situation. Said differ ently, no signaling is undertaken when the operator already identified a track as hos- 
tile. After an acquaintance period of one day, the WO performed three scenarios. Fig. 4 and table 1 summarize the average AT and the standard error of means for each scenario for one WO. These three scenarios served in the learning phase and the last column describes the outcome of the operator performance model by combining all three AT for the three scenarios. Accordingly, the OPM states that the operator should be aided by the CMS when the average response time reaches the threshold of 35.9 seconds (=32.9+ 3 seconds).

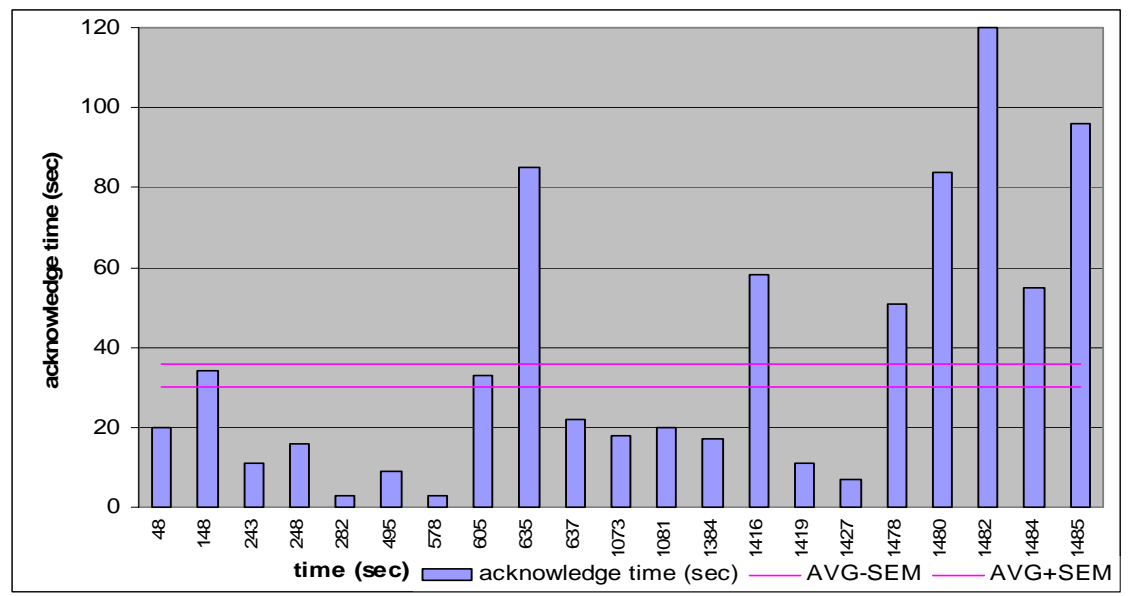

Fig. 5. The acknowledgement time and the OPM as a function of the second scenario

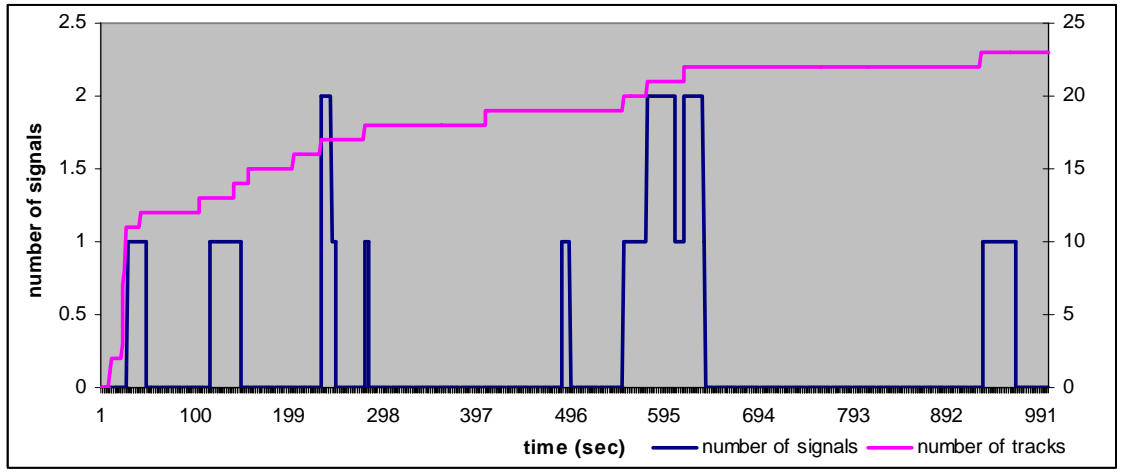

Fig. 6. The number of signals for the second scenario displays quiet areas

Fig. 5 demonstrates the acknowledgement times as a function of scenario two. The two horizontal lines illustrate the bandwidth of the model, i.e. the average plus or minus the SEM. Fig. 5 shows that for a total of 21 signals the operator requires in seven occasions more time to acknowledge a signal, and in twelve occasions the operator requires less time to acknowledge with respect to the OPM. At $t=148$ seconds and at 
$t=605$ seconds the mechanism is not triggering additional support of the system because the SEM absorbs slight fluctuation around the average. This seems beneficial in one case for not triggering, and another case $(t=605)$ it looks as if the mechanism should lead to a trigger. These results suggests that the OPM might facilitate triggering occasionally but, as stated by Scerbo [11], the model requires more information to become useful, and proactive.

\section{Task Load Model}

This section elaborates on environmental factors that lead to an expected increase of the workload. The current task load model (CTL) [15] is utilized for this purpose. The (CTL) model distinguishes three factors that have a substantial effect on the workload. The first factor, percentage time occupied (TO), has been used to assess workload in practice for time-line assessments. Such assessments are often based on the notion that people should not be occupied more than 70 to 80 percent of the total time available [16]. The second load factor is the level of information processing (LIP). To address the cognitive task demands, the cognitive load model incorporates the skillrule-knowledge framework of Rasmussen (1986) where the knowledge based component involves a high load on the limited capacity of working memory. To address the demands of attention shifts, the cognitive load model distinguishes task-set switching (TSS) as a third load factor. The tree factors present a three-dimensional space in which human activities can be projected with regions indicating the cognitive demands that the activity imposes on the operator. It should be noted that these factors represent task demands that affect the operator workload (i.e., it is not a definition of the operator cognitive state). Fig 7 shows from the perspective of the operational demands a number of expected cognitive states.

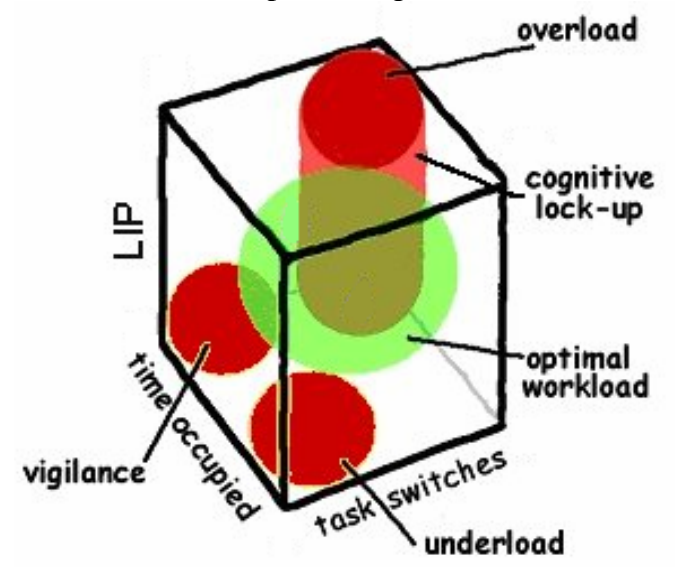

Fig. 7. The three dimensions of the task load model. Neerincx [14] distinguished several critical regions 
The workload cube may inspire us to find more workload indicators. First of all the number of information element, i.e. the tracks, may lead to an increase in the number of TS (switching between information elements for monitoring purposes) and TO. Fig 6 provides a nice example of this case in the timeframe $t=496$ untill $t=694$. The LIP, sometimes referred to as complexity, of the information element in C2 depends mainly on the identity (friend or foe) of a track. The identity often provides an intention, but sometimes the identity involves a lot of uncertainty. We state that uncertain elements lead to a higher LIP and table 1 provides an overview of the identities and expected LIP. For example, an increase in unknown tracks results in an increase in complexity since the operator has to put effort in the process to ascertain the identity of a track of which relatively little is known. The cognitive burden will be less when the same increase of track is labeled friendly.

Table 1. The expected level of information of an important feature of a track

\begin{tabular}{lll}
\hline Identity & Presumable intention & Expected LIP \\
\hline Pending & System is gathering information & Skill-based \\
\hline Unknown & Unknown & Knowledge-based \\
\hline Friendly & Track of own nation & Skill-based \\
\hline Assumed Friendly & Presumably own nation track & Knowledge-based \\
\hline Neutral & Track is commercial & Skill-based \\
\hline Suspect & Track might attack & Knowledge-based \\
\hline Hostile & Track might attack & Rule-based \\
\hline
\end{tabular}

\section{Workload Predictors}

Scerbo [11] mentions that a mitigation strategy should not be entirely reactive but preferably demonstrate a kind of pro-active behavior. This section describes an approach where such pro-activeness is achieved.

In C2, tracks are the predominant information elements and it is common that a number of operators split the surrounding world into sectors. Usually these sectors are divided geometrically (e.g. range), but not necessarily and the sectors could easily consist other attributes (e.g. speed, identity). Each operator is responsible for information elements in that sector, and the amount and identity of tracks provide an indicator of workload. The amount of information elements that is likely to enter the operator's sector in the following few minutes could therefore be an indicator of future workload, so pro-active adaptation could be based on estimates of this variable. The CMS uses the trajectories of the tracks to infer a velocity vector (see fig 8 ). This velocity vector is extrapolated into the future which provides the estimated number of tracks in the future hence an indication for future workload. 


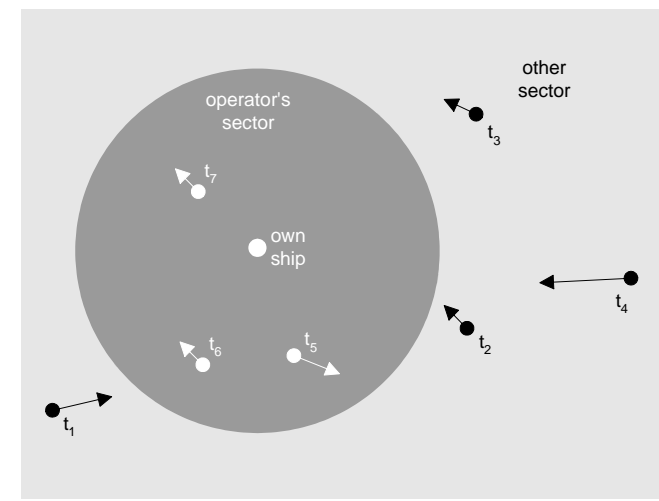

Fig. 8. Information elements that are not in the operational space may eventually enter

\section{Conclusion}

Technological developments enable crews of navy frigates to work more efficiently and effectively. These complex, autonomous, and information-rich environments creates a competition for the operator's attention between different information items, possibly leading to a cognitive overload. Limitations in human cognition are due to intrinsic restrictions and these may fluctuate from moment to moment depending on a host of factors including mental fatigue, novelty, boredom, and stress. The concept of adaptive automation promises a solution to the overwhelmed operator by shifting the amount of work between the human and the system in time, while maintaining a high level of situation awareness. One of the most critical challenges in developing adaptive human-machine collaboration concerns the design of a trigger mechanism. This paper discusses a performance model in the field of C2 and the proposed model hints towards a description of cognitive state. The evaluation shows that additional factors should be incorporated in the model. These are found by applying the cognitive task load model [15] to the field of C2 and a number of workload factors are identified. Also, it is suggested that triggering could be improved by a predictive measure.

This study demonstrates that the design of a trigger mechanism is difficult and that a number of factors need to be incorporated. We will continue research and evaluation along this path, and combine critical workload factors to an operator model.

\section{Acknowledgments}

CAMS-Force Vision, a software development company associated with the Royal Netherlands Navy, funded this research. 


\section{References}

1. Rouse, W. B.: Adaptive aiding for Human/Computer Control. Human factors (1988) 431443

2 Bailey, N. B., Scerbo, M. W., Freeman, F. G., Mikulka, P. J., Scott, A. S.: Comparison of a Brain-Based Adaptive System and a Manual Adaptable System for Invoking Automation. Human factors (2006) 693-709

3. Parasuraman, R., Mouloua, M., Molloy, R.: Effects of adaptive task allocation on monitoring of automated systems. Human factors (1996) 665-679

4. Kaber, D., Endlsey, M.: The effects of level of automation and adaptive automation on human performance, situation awareness and workload in a dynamic control task. Theroretical Issues in Ergonomics Science . (2004)

5. Hilburn, B., Jorna, P., Byrne, E., Parasuraman, R.: The effect of adaptive air traffic control (ATC) decisoin aiding on controller mental workload. In: Mouloua, M., Koonce, J. M. (eds): Human-automation interaction: research and practice. Lawrence Erlbaum Associates, Mahwah, NJ (1997) 84-91

6. Scallen, S., Hancock, P., Duley, J.: Pilot performance and preference for short cycles of automation in adaptive function allocation. Applied ergonomics (1995) 397-404

7. Inagaki, T.: Situation-adaptive Autonomy for Time-critical Takeoff Decisions. International journal of modelling and simulation (2000) 175-180

8. Moray, N., Inagaki, T., Itoh, M.: Adaptive Automation, Trust, and Self-Confidence in Fault Management of Time-Critical Tasks. Journal of experimental psychology (2000) 44-5714

9. Kruse, A. A., Schmorrow, D. D.: Session overview: Foundations of augmented cognition. In: Schmorrow, D. D. (ed.): Foundations of Augmented Cognition. Lawrence Erlbaum Associates, Mawah, NJ (2005) 441-445

10. Veltman, J.A., Jansen, C.: The role of operator state assessment in adaptive automation. (2006), TNO Human Factors Research Institute.

11. Scerbo, M.: Theoretical perspectives on adaptive automation. In: Parasuraman, R., Mouloua, M. (eds): Automation and human performance: theory and applications. Lawrence Erlbaum Assiciated, Publishes Mahwah, New Jersey (1996) 37-63

12. Scerbo, M.W., Freeman, F G, Mikulka, P J, Parasuraman, R, Nocero, F D, Prinzel, L J.: The Efficacy of Psychophysiological. NASA/TP-2001-211018. (2001)

13. Shannon, C.,.Weaver, W.: The mathematical theory of communications. University of Illinois Press, Urbana. (1949)

14. van Delft, J.H., Arciszewski, H F R.: The evaluation of automation and support tools in the naval command \& control environment. TM-04-A067. (2004) Soesterberg, The Netherlands, TNO Human Factors.

15. Neerincx, M. A.: Cognitive task load design: model, methods and examples. In: Hollnagel, E. (ed.): Handbook of Cognitive Task Design. Lawrence Erlbaum Associates, Mahwah, NJ (2003) 283-305

16. Beevis, D.: Analysis techniques for man-machine systems design, Vol 1 \& 2. NATO/Panel 8-RSG.14, Technical Report AC/243(Panel 8)TR/7. (1992) Brussels: North Atlantic treaty organization. 